\title{
The influence of a clinical classification system on the management of patients with positional dependent obstructive sleep apnoea
}

\author{
Sophia E. Schiza • Charalampos Mermigkis • \\ Izolde Bouloukaki
}

Received: 24 June 2014 / Revised: 3 July 2014 / Accepted: 8 July 2014 /Published online: 15 July 2014

(C) Springer-Verlag Berlin Heidelberg 2014

Obstructive sleep apnoea syndrome (OSAS) is a common disease with significant morbidity, which treatment depends in part on the severity of the disease. The incidence of sleepdisordered breathing events, in many patients, is substantially greater during sleep in the supine position than in the lateral recumbent position. Upper airway function is typically more compromised in the supine posture, due to unfavourable gravitational effects leading to an increased propensity for and frequency of upper airway collapse, higher airway opening pressures and more prolonged and severe respiratory events with greater oxygen desaturation. Therefore, sleeping position is a clinically relevant parameter that must be taken into account when dealing with several sleep disturbances.

The detrimental effect of the supine position on the severity of the snoring or apnoea was probably first identified, many years ago, by the spouses of habitual snorers and OSAS patients. However, it was only in 1984 when Cartwright suggested that OSAS patients could be divided into positional, traditionally defined as those OSAS patients in whom the apnoea-hypopnoea index (AHI) was at least twice as high in the supine position as in the lateral position and non-positional patients [1]. This is the most common definition for positional obstructive sleep apnoea (POSA) used today. Positional OSA patients who meet the traditional criteria have a prevalence of 50 to $60 \%$, a high percentage that cannot be ignored. Since then, Cartwright's criteria are questioned leading to creation and application of adapted versions, such as the one of Marklund et al. in 1998 [2], who defined POSA as supine apnoea-hypopnoea index $\geq 10$, together with a lateral apnoea-

S. E. Schiza $(\bowtie) \cdot$ C. Mermigkis $\cdot$ I. Bouloukaki

Sleep Disorders Center, Department of Thoracic Medicine,

University General Hospital, Medical School of the University

of Crete, 71110 Heraklion, Crete, Greece

e-mail: schiza@med.uoc.gr hypopnoea index $<10$. A more stringent definition by Mador et al. requires supine AHI at least twice the lateral AHI, but with a lateral AHI not exceeding five [3]. Using this possibly therapeutically more relevant definition, a high prevalence of positional OSAS ( $27 \%$ overall) was also reported, with $50 \%$ of patients with mild OSA and $19 \%$ of those with moderate OSAS having positional OSA. More recently, Bignold et al. [4], examining the prevalence of positional OSAS, introduced a minimum sleeping time spent in different positions, using a definition of an overall $\mathrm{AHI} \geq 15 / \mathrm{h}$, supine $\mathrm{AHI} \geq$ twice the non-supine $\mathrm{AHI} \geq 20 \mathrm{~min}$ of sleep in supine and non-supine postures and non-supine $\mathrm{AHI}<15$.

In general, the prevalence of positional patients among those with OSAS varies from 9 to $60 \%$, showing a higher prevalence in Asians than in Whites [5]. Furthermore, positional dependency is inversely related to OSAS severity, BMI and age $[6,7]$. Therefore, as the prevalence of POSA is higher in mild-moderate OSA, mild OSA patients, especially the less symptomatic ones, may be good candidates for positional therapy (PT). It is reasonable to speculate that this form of therapy could benefit a considerable number of patients with OSAS because mild OSAS patients are the vast majority of OSAS patients [8].

The retrospective study by Dr. Ravesloot et al. tried to identify patients that will benefit from PT, by creating a new classification system for POSA, the Amsterdam Positional OSAS Classification "APOC criteria", which includes the total sleep time (TST) spent in different sleep positions. The classification attempts to identify those patients who could truly benefit from PT alone versus those for whom PT would be an adjunct therapy to improve results of other treatment modalities. The study was performed on 100 randomly selected patients from a database. According to these criteria, $55 \%$ of the patients were diagnosed with POSA. The authors conclude that based on their data, the APOC criteria for POSA are 
more effective compared to previously applied POSA criteria, in identifying patients that will benefit from PT. This study has certain clinical importance as the majority of previous POSA classification systems do not take into account the TST spent in different positions, but use the differential advantage of the non-supine AHI over the supine AHI calculated as a ratio. Using this approach in POSA classification, cases with positional OSAS should be evaluated as a different clinical entity and treatment plan should be made accordingly. Furthermore this study adds important information to our knowledge on this topic and highlights the many gaps that we still need to fill up in order to understand the real therapeutic value of the PT for POSA patients. Researchers should keep in mind the criteria, till now, used to evaluate POSA when critically evaluating published reports describing the effects of therapeutic interventions for POSA.

Identifying patients with OSAS as being either positional or non-positional has important therapeutic implications. This distinction is essential since only patients who show most of their breathing abnormalities while sleeping in the supine position may substantially benefit from PT. For these patients, the critical issue is that the severity of the disease is totally dependent on the sleep time spent or not spent in the supine posture. Obviously, PT is not an adequate option for nonPOSA patients who continue to show a high amount of breathing abnormalities in non-supine postures and as a consequence for those patients, continuous positive airway pressure (CPAP) is the treatment of choice.

Nevertheless, it is also important to recognize that findings of polysomnography (PSG) studies should be interpreted with caution, as the time spent in supine position during a PSG study may not be an accurate reflection of the time spent in supine position during sleep at home. Data suggest that patients spend more time in the supine posture, as grater as $56 \%$, during PSG study compared to home sleep. Therefore, encouraging supine sleep, due to the routine practice in clinical studies, the time spent in supine position during sleep maybe overestimated compared to home sleep patterns. As a consequence, the severity of OSAS may be overestimated as well, considering that the overall AHI is affected by the relative proportion of each of the different sleeping positions. Furthermore, reliance on a single PSG night continues to represent a challenge to OSAS diagnosis, because a single night of sleep often does not provide sufficient examination of all combinations of supine/non-supine sleep. Despite that, studies have found similar mean AHI values across study nights and good test-retest correlations. Keeping this in mind, sleep study centers need to recognize that and advise their patients to occupy their natural sleep postures during the PSG study.

One of the oldest interventions for snoring and OSAS was the bed partner's prompting to move to the lateral recumbent position. Forcing a change to the non-supine position during sleep can be an effective treatment. Positional therapy can be defined as preventing patients from sleeping in the worst sleeping position, which is usually, but not always, the supine position. This appears to be an adequate form of therapy for positional patients if there is sufficient control of OSAS, as well as maintenance of acceptable oxygenation and sleep continuity, in the lateral recumbent position during REM and NREM sleep. Some patients with POSA have succeeded in lowering their total AHI to normal by merely sleeping on their sides and it has been estimated that this type of therapy alone could be successful. This positional effect seen in sleep apnoea led to development of techniques that encourage patients to sleep on their sides. PT, which is often simple and inexpensive, may constitute as a stand-alone treatment or as an additional measure to increase the success rate of other established treatment methods. Studies report a positive effect of PT on the AHI, and a better compliance than CPAP compliance, although the latter is a more effective treatment, as it can further reduce the severity of sleep apnoea in AHI and also increase oxygen saturation level [9]. Moreover, long-term compliance for PT remains an issue, due to ineffectiveness, backache, discomfort and no improvement in sleep quality or daytime alertness, leading to a serious lack of compliance [10]. Remarkable results using innovative treatment concepts for PT have been reported; still, there is room for both technical improvement of the devices and for further research.

In conclusion, the influence of body position on upper airway patency during sleep is a well-known phenomenon and the high prevalence of POSA implies that the amount of supine sleep time is influential in determining the degree of OSAS in a majority of subjects. Therefore, clinicians should question specifically the patient and bed partner regarding position dependence of sleep-disordered breathing and objectively evaluate sleep and breathing in these different body positions. In spite of the absence of a universal standard definition, patients with POSA are more likely to benefit from positional therapy, leading to the clinically significant improvement of OSAS in a subset of patients. Further understanding of postural influence on the severity of sleep apnoea may help in identifying therapeutically more favourable interventions.

\section{References}

1. Cartwright RD (1984) Effect of sleep position on sleep apnea severity. Sleep 7:110-114

2. Marklund M, Persson M, Franklin KA (1998) Treatment success with a mandibular advancement device is related to supinedependent sleep apnea. Chest 114:1630-1635

3. Mador MJ, Kufel TJ, Magalang UJ, Rajesh SK, Watwe V, Grant BJ (2005) Prevalence of positional sleep apnea in patients undergoing polysomnography. Chest 128:2130-2137

4. Bignold JJ, Mercer JD, Antic NA, McEvoy RD, Catcheside PG (2011) Accurate position monitoring and improved supine dependent 
obstructive sleep apnea with a new position recording and supine avoidance device. J Clin Sleep Med 7:376-383

5. Teerapraipruk B, Chirakalwasan N, Simon R, Hirunwiwatkul P, Jaimchariyatam N, Desudchit T, Charakorn N, Wanlapakorn C (2012) Clinical and polysomnographic data of positional sleep apnea and its predictors. Sleep Breath 16:1167-1172

6. Oksenberg A, Silverberg DS, Arons E, Radwan H (1997) Positional vs nonpositional obstructive sleep apnea patients: anthropomorphic, nocturnal polysomnographic, and multiple sleep latency test data. Chest 112:629-639

7. Sunwoo WS, Hong SL, Kim SW, Park SJ, Han DH, Kim JW, Lee CH, Rhee CS (2012) Association between positional dependency and obstruction site in obstructive sleep apnea syndrome. Clin Exp Otorhinolaryngol 5:218-221

8. Young T, Peppard PE, Gottlieb DJ (2002) Epidemiology of obstructive sleep apnea. Am J Respir Crit Care Med 165:1217-1239

9. Ha SC, Hirai HW, Tsoi KK (2014) Comparison of positional therapy versus continuous positive airway pressure in patients with positional obstructive sleep apnea: a meta-analysis of randomized trials. Sleep Med Rev 18:19-24

10. Ravesloot MJ, van Maanen JP, Dun L, de Vries N (2013) The undervalued potential of positional therapy in position dependent snoring and obstructive sleep apnea-a review of the literature. Sleep Breath 17:39-49 